\title{
Teorias clássicas de calibre como sistemas com vínculos: um ponto de vista geométrico
}

Classical gauge theories as systems with constraints: a geometric point of view

\author{
M. F. Araujo de Resende*1 \\ ${ }^{1}$ Instituto de Física, Universidade de São Paulo, 05508-090 São Paulo SP, Brasil
}

Recebido em 08 de Fevereiro, 2017. Revisado em 14 de Julho, 2017. Aceito em 14 de Julho, 2017.

\begin{abstract}
Neste trabalho fazemos uma breve revisão sobre a formulação hamiltoniana dos sistemas clássicos que estão vinculados a subvariedades para que, dentro deste contexto, fique claro qual é o verdadeiro significado de uma teoria clássica de calibre.

Palavras-chave: sistemas clássicos, variedades diferenciáveis, teorias de calibre.
\end{abstract}

In this work we make a brief review on the Hamiltonian formulation of classical systems that are constrained to submanifolds so that, within this context, make it clear what is the true meaning of a classical gauge theory. Keywords: classical systems, differentiable manifolds, gauge theories.

\section{Introdução}

De acordo com a literatura, diversas teorias físicas podem ser classificadas como teorias de calibre, entre as quais podemos listar alguns exemplos bem famosos, como a Eletrodinâmica Clássica [1] e o Modelo Padrão das partículas elementares $[2]$. Aliás, no que diz respeito à Eletrodinâmica Clássica, por exemplo, vale dizer que ela é muito mais do que um exemplo famoso, pois é através dela que o conceito de uma teoria de calibre normalmente é introduzido aos estudantes. Essa introdução é feita sob a afirmação de que as equações de movimento dessa teoria são covariantes: ou seja, quando os campos elétrico e magnético não variam quando realizamos alterações específicas nos potenciais que os definem [3]. Isso se deve ao fato de que esses campos são definidos por derivadas dos potenciais. Logo, para mantê-los invariantes, qualquer transformação que seja anulada por essas derivadas será válida.

Apesar de não ser difícil mostrar a invariância desses campos, e consequentemente das equações de movimento de uma teoria de calibre após essas "alterações específicas", algo que ainda não está muito claro para algumas pessoas é o que é realmente uma teoria de calibre. Aliás, por que uma teoria de calibre leva esse nome? Será que existe algo mais fundamental por trás do fato das equações de movimento de um sistema físico continuarem sendo expressas com o mesmo formato mesmo depois destas alterações?

Diante de todas essas possíveis indagações, o objetivo do nosso trabalho é um só: oferecer ao leitor um texto (que tenta ser o mais didático possível dentro dos padrões

*Endereço de correspondência: resende@if.usp.br de um artigo científico) com uma interpretação relativamente simples, porém bastante fundamental, que visa esclarecer o que é uma teoria clássica de calibre. Fazemos isso tomando como base um fato que não costuma ser apresentado na literatura comum desse tema: que uma teoria clássica de calibre é aquela que descreve um sistema físico que está vinculado a uma subvariedade que pode ser fixada de infinitas maneiras.

Para deixar claro ao leitor o que esta nossa última afirmação quer dizer, cada uma das Seções deste trabalho será dedicada a um tópico específico. No caso da próxima Seção, fazemos uma apresentação básica sobre a descrição de sistemas clássicos, mostrando porque é razoável supor, por exemplo, que os espaços onde estes sistemas estão definidos se identificam como variedades diferenciáveis. Já a Seção 3 é dedicada à apresentação do que é a teoria de sistemas clássicos com vínculos sob a adoção de uma formulação hamiltoniana, para que, na Seção 4, uma teoria clássica de calibre possa ser definida como um caso particular das teorias com vínculos. Por fim, vale dizer que, além de "salpicarmos" estas Seções com exemplos que são úteis tanto à compreensão dos sistemas com vínculos como das teorias clássicas de calibre, usamos a Subseção 4.3 para ilustrar como essa interpretação, de uma teoria clássica calibre como um sistema hamiltoniano com vínculos, ajusta-se a um exemplo tradicional da Eletrodinâmica Clássica.

\section{Sobre a descrição de sistemas clássicos}

Quando precisamos descrever um sistema clássico que está definido num espaço com $n$ dimensões, sempre procuramos fazer isso da maneira mais simples possível, 
associando a função $x^{j}:[0, \infty) \rightarrow \mathbb{R}$ que melhor se ajusta à trajetória do sistema na $j$-ésima direção, onde $j=1, \ldots, n$. Entretanto, existem, pelo menos, dois bons motivos para afirmarmos que fazer tal ajuste nem sempre é algo tão simples. O primeiro deles pode ser bem entendido desde que notemos que, como um espaço $n$ dimensional pode ser parametrizado por mais de um sistema de coordenadas, este ajuste de funções pode ser feito de modo não único. É exatamente isso o que acontece, por exemplo, com um sistema físico que está definido sobre uma esfera de raio $R$ constante que está mergulhada no $\mathbb{R}^{3}$ : afinal, apesar de ser perfeitamente possível descrever esse sistema por meio de uma função

$$
x(t)=\left(x^{1}(t), x^{2}(t), x^{3}(t)\right)
$$

que se apoia sobre uma perspectiva cartesiana do $\mathbb{R}^{3}$, também é perfeitamente possível descrevê-lo usando uma outra função

$$
q(t)=(\phi(t), \theta(t), R)
$$

que se vale de uma perspectiva esférica.

Apesar de, neste exemplo, parecer que a descrição que é feita através de uma função $q:[0, \infty) \rightarrow \mathbb{R}^{3}$ é mais confortável (já que ela se vale de um número menor de funções não constantes quando comparada a $x:[0, \infty) \rightarrow \mathbb{R}^{3}$ ), vale observar que as situações físicas reais nem sempre são tão simples como a de um sistema que está definido sobre uma esfera. E, no caso, é exatamente diante desta observação que surge o segundo motivo que nos faz afirmar que fazer tal ajuste de funções nem sempre é fácil: pois 4],

- se, por um lado, nem sempre somos capazes de enxergar todas as simetrias que um sistema físico possui (as quais certamente nos levariam a uma descrição mais simples, haja vista que elas reduziriam o número de parâmetros independentes envolvidos para com esta descrição),

- por outro lado, as formulações lagrangiana e hamiltoniana que descrevem um sistema nem sempre nos levam a um conjunto de equações mais facilmente resolvível, se expressas em termos de um número menor de parâmetros ou de funções não constantes.

Além desses dois motivos, se também quisermos apontar um terceiro, este pode perfeitamente se relacionar, por exemplo, com a diferenciabilidade das funções que são ajustadas às trajetórias do sistema. Porém, quando supomos que essas trajetórias podem ser modeladas por funções que são diferenciáveis ${ }^{1}$ até uma ordem $k$, passa a ser perfeitamente válido considerar que todas as posições que o sistema assume pertencem a uma variedade diferenciável; ou seja, que estas posições pertencem a um espaço topológico $\mathcal{M}_{n}$ que não é necessariamente plano,

\footnotetext{
${ }^{1}$ Suposição que é justamente a que fazemos quando, a partir da função que modela a aceleração, obtemos as equações de movimento de um sistema.
}

que tem $n$ dimensões e que pode ser interpretado como uma generalização de um espaço euclidiano [5].

A propósito, é devido a toda essa questão da diferenciabilidade de uma variedade $\AA^{2}$ que acaba ficando claro que, para todas as curvas que podem ser definidas através de aplicações diferenciáveis $\alpha:(-\varepsilon, \varepsilon) \rightarrow \mathcal{M}_{n}$, também somos capazes de definir vetores que são tangentes a elas em cada um dos seus pontos [6]. Trata-se de uma observação que, apesar de simples, é muito importante ao contexto da Mecânica Clássica, haja vista que é esta observação quem nos mostra que, quando analisamos um sistema clássico usando uma formulação lagrangiana, a sua descrição se dá através de uma função $L: T \mathcal{M}_{n} \rightarrow \mathbb{R}$ cujo domínio

$$
T \mathcal{M}_{n}=\left\{(x, \dot{x}): x \in \mathcal{M}_{n}, \dot{x} \in T_{x} \mathcal{M}_{n}\right\}
$$

se identifica como um fibrado tangente [6]; ou seja, um domínio que se identifica como um conjunto que, por ser definido como a união disjunta de todos os espaços $T_{x} \mathcal{M}_{n}$ que são tangentes em cada ponto de $\mathcal{M}_{n}$, é capaz de nos dar informações sobre a posição $x=\left(x^{1}, \ldots, x^{n}\right)$ e a velocidade $\dot{x}=\left(\dot{x}^{1}, \ldots, \dot{x}^{n}\right)$ do sistema. Tais informações são imprescindíveis para a determinação unívoca da trajetória desse sistema sobre $\mathcal{M}_{n}$ entre os instantes $t_{1}$ e $t_{2}$, a qual pode ser obtida das equações de Euler-Lagrange

$$
\frac{\delta S}{\delta x^{j}}=\frac{\partial L}{\partial x^{j}}-\frac{d}{d t} \frac{\partial L}{\partial \dot{x}^{j}}=0
$$

desde que admitamos que a $a c ̧ \tilde{a} o$

$$
S=\int_{t_{1}}^{t_{2}} L d t
$$

relacionada ao sistema seja estacionária 7].

\section{Teorias de sistemas com vínculos}

Em todo caso, vale dizer que existe um bom motivo geométrico para darmos destaque à identificação do domínio de $L$ como um fibrado tangente, motivo esse que não se atém ao jargão matemático. Para entender qual é esse motivo, devemos levar em conta que a expressão da energia do sistema em moldes lagrangianos é dada por 8

$$
\mathcal{E}=\frac{\partial L}{\partial \dot{x}^{j}} \dot{x}^{j}-L
$$

Pois, como é a partir desta expressão que a função que protagoniza a formulação hamiltoniana de um sistema clássico se constroi como

$$
H=\left.\mathcal{E}\right|_{\dot{x}=\bar{v}(q, P)},
$$

é justamente isso que mostra que o domínio (ou espaço de fase) de $H$ é um fibrado cotangente $T^{*} \mathcal{M}_{n}$; ou seja,

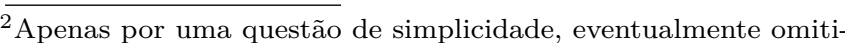
remos o termo "diferenciável" que predica uma variedade, assim como também omitiremos que essa diferenciabilidade só vai até uma ordem $k$.
} 
o domínio de $H$ é a união disjunta de todos os espaços que são cotangentes em cada ponto de $\mathcal{M}_{n}$, já que a transformação

$$
P_{j}:=\frac{\partial L}{\partial \dot{x}^{j}}
$$

que o define troca os parâmetros $\dot{x}^{j}$ pelos seus duais $P_{j}$.

Do ponto de vista mais básico, que está relacionado à transição entre essas duas formulações (e o qual também consta em grande parte dos livros que tratam de Mecânica Clássica), é possível afirmar que a única diferença prática que existe entre elas é que

- enquanto, na primeira (que é a lagrangiana), o sistema clássico é descrito por um conjunto de $n$ equações (1) que contém derivadas de segunda ordem,

- na segunda (que é a hamiltoniana) usamos um conjunto de $2 n$ equações

$$
\dot{x}^{j}=\frac{\partial H}{\partial P_{j}} \quad \text { e } \quad \dot{P}_{j}=-\frac{\partial H}{\partial x^{j}}
$$

equivalente a (1) para descrever a mesma dinâmica, agora em termos das posições $x^{j}$ e dos momentos $P_{j}$, só que usando apenas derivadas de primeira ordem 9 .

Em outras palavras, as equações de movimento de um sistema clássico até podem se tornar mais fáceis de serem resolvidas quando escritas sob uma formulação hamiltoniana, porém dobram de tamanho. No entanto, do ponto de vista geométrico que permeia essas duas formulações, uma das coisas que também podem ser ditas é que, no que diz respeito à definição dos domínios de $L$ e $H$, tanto faz se lidarmos com uma formulação ou com outra: como estamos assumindo que as posições do sistema pertencem a uma variedade $\mathcal{M}_{n}$, esses dois domínios sempre se identificam como duas variedades e, portanto, podem ser parametrizados de infinitas maneiras [6]. Embora, por enquanto, essa informação possa soar como uma mera "curiosidade matemática", ela mostrará toda a sua relevância um pouco mais adiante, mais especificamente quando apresentarmos a definição do que é uma teoria clássica de calibre na Subseção 4.2

Contudo, antes de dizermos qual é essa "informação relevante", já existe uma observação a ser feita sobre a transformação (3) que, inclusive, também será bastante útil quando tal "informação relevante" for dita. Afinal de contas, existem funções lagrangianas $L$ onde, por exemplo, alguns momentos podem ser nulos. E como o teorema da função inversa [10] nos diz que, quando $P_{a}=0$, não é possível expressar $\dot{x}^{a}$ como uma função $v^{a}(x, P)$, é justamente esta nulidade que nos mostra não apenas que $P_{a}$ é uma constante de movimento: esta nulidade também nos mostra que os parâmetros que descrevem o sistema não são independentes. Ou seja, pelo mesmo ponto de vista geométrico que já usamos acima, é válido afirmar que o sistema físico que essa teoria clássica descreve está vinculado a um subconjunto $T^{*} \mathcal{M}_{n-m} \subset T^{*} \mathcal{M}_{n}$ que é, no mínimo, definido por

$$
\Phi_{1}(x, P)=P_{a}=0 .
$$

Quando esse é o caso, este vínculo (4) deve ser incorporado à função $H: T^{*} \mathcal{M}_{n} \rightarrow \mathbb{R}$ através de um multiplicador de lagrange $\lambda^{1}$ que passa a ser visto como um novo parâmetro do sistema, o que leva a uma hamiltoniana primária

$$
H^{(1)}=H+\lambda^{1} \Phi_{1}
$$

capaz de descrever o sistema físico apenas quando $\Phi_{1}=0$.

\subsection{Equações de regularidade}

Embora (4) seja um vínculo que define o sistema físico sob consideração, como nada garante que este vínculo seja o único, passa a ser fundamental verificar se existem outros vínculos definindo esse subconjunto $T^{*} \mathcal{M}_{n-m}$ que, no caso, deve ser interpretado como uma subvariedade; ou seja, $T^{*} \mathcal{M}_{n-m}$ é um subconjunto que pode ser visto como uma superfíci $\varrho^{3}$ que está mergulhada em $T^{*} \mathcal{M}_{n}$. Assim, notando que o sistema físico já satisfaz a (4), a melhor maneira de fazer tal verificação é usar, como ponto de partida, o fato de que

$$
\Phi_{1}=0 \Rightarrow \dot{\Phi}_{1}=0
$$

é uma relação válida na superfície em questão. Pois, se $\Phi_{1}=0$ é uma relação que é imutável por definir uma parte desta superfície, $\dot{\Phi}_{1}=0$ também será.

\subsubsection{Parênteses de Poisson e o algoritmo de Dirac-Bergmann}

Diante dessa necessidade de avaliar o que mais pode surgir de (6), não deixa de ser interessante notar que toda a dinâmica do sistema em questão pode ser expressa de uma forma bastante simples usando os chamados parênteses de Poisson [7] que, para duas funções $F, G: T^{*} \mathcal{M}_{n} \rightarrow \mathbb{R}$, são definidos por

$$
\{F, G\}=\frac{\partial F}{\partial x^{j}} \frac{\partial G}{\partial P_{j}}-\frac{\partial F}{\partial P_{j}} \frac{\partial G}{\partial x^{j}} .
$$

Pois, além deles nos permitirem escrever todas as equações de movimento

$$
\dot{z}=\left\{z, H^{(1)}\right\}
$$

de uma forma um pouco mais "enxuta", sob a consideração de que $z=(x, P)$, eles também nos mostram que qualquer vínculo $\Phi_{1}=0$, que define a subvariedade $T^{*} \mathcal{M}_{n-m}$ onde o sistema físico está definido, também satisfaz uma equação

$$
\dot{\Phi}_{1}=\left\{\Phi_{1}, H^{(1)}\right\}=0
$$

que só vale nesta subvariedade. Ou seja, se quisermos avaliar se o sistema possui outra constante de movimento

\footnotetext{
${ }^{3} \mathrm{Na}$ verdade, como uma hipersuperfície quando $n-m>2$.
} 
$\Phi_{2}=0$ além de $\Phi_{1}=0$, basta calcular $\left\{\Phi_{1}, H^{(1)}\right\}$ e igualar o resultado a zero: se o que surgir disso for uma aplicação $\Phi_{2}: T^{*} \mathcal{M}_{n} \rightarrow \mathbb{R}$ independente da anterior, ela deve ser incorporada a (5) para que uma nova rodada de avaliação seja feita, usando uma nova hamiltoniana

$$
H^{(2)}=H^{(1)}+\lambda^{2} \Phi_{2}
$$

que é um pouco mais completa que $H^{(1)}$ no que diz respeito à descrição do sistema. Se, ao final desse processo de investigação e de incorporação de vínculos, da equação de regularidade

$$
\dot{\Phi}_{m}=\left\{\Phi_{m}, H^{(m)}\right\}=0,
$$

que precisa ser válida na subvariedade $T^{*} \mathcal{M}_{n-m}$, não surgir mais nenhuma aplicação $\Phi_{m+1}: T^{*} \mathcal{M}_{n} \rightarrow \mathbb{R}$ independente das anteriores $\Phi_{1}, \ldots, \Phi_{m}$, a dinâmica do sistema fica completamente definida através de

$$
\dot{z}=\left\{z, H_{\mathrm{T}}\right\}
$$

Aqui,

$$
H_{\mathrm{T}}=H^{(m)}=H+\lambda^{a} \Phi_{a}, \quad \text { com } \quad a=1, \ldots, m,
$$

é a hamiltoniana total do sistema, cuja física se restringe apenas à subvariedade $T^{*} \mathcal{M}_{n-m}$ que é definida por

$$
\Phi(z)=\left(\Phi_{1}(z), \ldots, \Phi_{m}(z)\right)=(0, \ldots, 0) .
$$

Este procedimento de procura de vínculos é conhecido na literatura como o algoritmo de Dirac-Bergmann [11.

\subsection{Uma observação importante}

Embora o que iremos dizer agora vá parecer algo tão técnico quanto os termos "fibrado tangente" e "fibrado cotangente" que mencionamos há algumas linhas, para o bom entendimento do que segue é importante destacar que, de acordo com P. A. M. Dirac [11]:

- uma função $\mathcal{F}: T^{*} \mathcal{M}_{n} \rightarrow \mathbb{R}$ é de primeira classe quando, para todo índice $a$, temos $\left\{\mathcal{F}, \Phi_{a}\right\}=0$ na subvariedade $T^{*} \mathcal{M}_{n-m}$;

- caso contrário, $\mathcal{F}$ é uma função de segunda classe.

Apesar desta aparente "tecnicalidade", o principal motivo que nos faz dizê-la é que, como qualquer $\Phi_{a}$ é um exemplo destas funções, quando consideramos que $\Theta$ é uma matriz quadrada de ordem $m$ com elementos dados por

$$
\Theta_{a b}=\left\{\Phi_{a}, \Phi_{b}\right\},
$$

é possível observar uma igualdade entre o posto $K$ desta matriz ${ }^{4}$ e o número de vínculos de segunda classe que definem a nossa teoria. Por consequência, se $\Theta$ for singular 5 (i) além disso implicar que $m-K$ vínculos serão de

${ }^{4} \mathrm{Ou}$ seja, o número $K$ de linhas ou colunas que são linearmente independentes em $\Theta[12$.

${ }^{5}$ Isto é, se $\Theta$ for uma matriz cujo determinante é igual a zero. primeira classe [8], (ii) como das equações de regularidade (9) segue que

$$
\dot{\Phi}_{a}=\left\{\Phi_{a}, H_{\mathrm{T}}\right\}=\left\{\Phi_{a}, H\right\}+\left\{\Phi_{a}, \Phi_{b}\right\} \lambda^{b}=0,
$$

isso mostra que os $m-K$ multiplicadores de Lagrange (que são usados para implementar estes $m-K$ vínculos de primeira classe a $H_{\mathrm{T}}$ ) não poderão ser resolvidos univocamente. Como se tornará claro logo a seguir, é exatamente por trás desta não univocidade que repousa a interpretação de uma teoria de calibre.

\section{Teorias clássicas de calibre}

\subsection{Um primeiro exemplo}

Para introduzir o leitor à ideia de como uma teoria clássica de calibre pode ser interpretada como um caso particular das teorias de sistemas com vínculos, começaremos analisando um exemplo simples: vamos considerar um sistema clássico que é modelado pela lagrangiana 8]

$$
L=\frac{1}{2}(\dot{x}-y)^{2} \text {. }
$$

Afinal, uma das vantagens deste exemplo é que, a partir das suas equações de movimento

$$
\frac{\delta S}{\delta x}=\dot{y}-\ddot{x}=0 \quad \text { e } \quad \frac{\delta S}{\delta y}=y-\dot{x}=0,
$$

já fica claro que existe uma restrição (ou seja, um vínculo) entre o comportamento de $y(t)$ e de $\dot{x}(t)$.

Diga-se de passagem, vale notar que essa mesma conclusão vinculativa, que transparece em 15 sob moldes lagrangianos, também transparece naturalmente quando adotamos uma formulação hamiltoniana, haja vista que, seja qual for a formulação que usamos para a analisar um sistema, ele continua sendo o mesmo: basta ver que, além das relações

$$
P_{x}=\frac{\partial L}{\partial \dot{x}}=\dot{x}-y \quad \text { e } \quad P_{y}=0
$$

nos mostrarem que

$$
\Phi_{1}=P_{y}=0
$$

deve ser interpretado como o vínculo primário do sistema em questão, é a partir da hamiltoniana primária

$$
H^{(1)}=H+\lambda^{1} \Phi_{1}
$$

(que é obtida segundo a prescrição feita na última Seção), onde

$$
H=\frac{1}{2} P_{x}^{2}+y P_{x},
$$

que a relação $y-\dot{x}=0$ acaba "ressurgindo" como um vínculo secundário do sistema, haja vista que

$$
\dot{\Phi}_{1}=\left\{\Phi_{1}, H^{(1)}\right\}=\left\{P_{y}, y\right\} P_{x}=0 \Rightarrow \Phi_{2}=P_{x}=0 .
$$


Em todo caso, devido à simplicidade desses resultados, algo que certamente o leitor pode estar se perguntando é sobre o porquê deste exemplo específico estar sendo usado para introduzir a ideia de uma teoria clássica de calibre. E a melhor resposta que podemos dar ao leitor que se faz esta pergunta se divide em duas partes onde, a primeira delas, pode ser bem entendida desde que ele note que nada de novo surge de

$$
\dot{\Phi}_{2}=\left\{\Phi_{2}, H^{(2)}\right\}=0 \text {. }
$$

Ou seja, como esta equação de regularidade é identicamente nula para uma hamiltoniana secundária

$$
H^{(2)}=H^{(1)}+\lambda^{2} \Phi_{2}=\frac{1}{2} P_{x}^{2}+\lambda_{1} P_{y}+\left(y+\lambda_{2}\right) P_{x}
$$

que é um pouco mais "refinada" que a 16 , os únicos vínculos aos quais o sistema está submisso são

$$
\Phi_{1}=P_{y}=0 \quad \text { e } \quad \Phi_{2}=P_{x}=0 .
$$

E a grande virtude desta conclusão não é nem que estes são os dois únicos vínculos do sistema físico: a grande virtude desta conclusão é que, como

$$
\left\{\Phi_{1}, \Phi_{2}\right\}=\left\{P_{y}, P_{x}\right\}=0
$$

é exatamente isso que caracteriza estes dois vínculos como de primeira classe.

Nestes termos, como já sugerimos ao final da última Seção que a presença de vínculos de primeira classe está diretamente relacionada à caracterização de uma teoria como "de calibre", acaba ficando claro o porquê de termos usado esse sistema, que é modelado por (14), como um exemplo, embora (i) ele seja apenas um entre vários e (ii) ainda não explicamos como essa presença de vínculos de primeira classe caracteriza uma teoria clássica de calibre. Esse último ponto será explicado apenas na Subseção 4.2

\subsubsection{Sobre a interpretação usual de uma teoria de calibre}

Entretanto, é quando olhamos para as equações de movimento 156 que a segunda parte da melhor resposta que podemos dar ao leitor aparece. Afinal, devemos notar que, como a dinâmica do sistema em pauta deve ser tal que $y-\dot{x}=0$, as condições iniciais de contorno

$$
x(0)=\alpha \quad, \quad \dot{x}(0)=y(0)=\beta \quad \text { e } \quad \dot{y}(0)=\gamma
$$

(onde $\alpha, \beta$ e $\gamma$ são três constantes reais) nos levam à solução

$$
x(t)=\alpha+\beta t+\frac{\gamma}{2} t^{2}+\int_{0}^{t} \varphi\left(t^{\prime}\right) d t^{\prime} \quad \text { e } \quad y(t)=\beta+\gamma t+\varphi(t)
$$

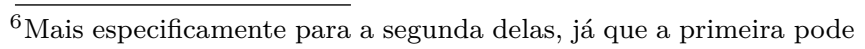
ser vista como um corolário da segunda. mais geral possível de 15. Aqui, $\varphi(t)$ é uma função tal que $\varphi(t)=\dot{\varphi}(t)=0$.

Diante deste resultado, e do fato de 15 também nos mostrar que 8

$$
\left(\frac{\delta}{\delta x}-\frac{d}{d t} \frac{\delta}{\delta y}\right) S=0
$$

vemos que tanto a ação $S$ como a lagrangiana $L$ são invariantes sob as transformações

$$
x \rightarrow x^{\prime}=x+f \quad \text { e } \quad y \quad \rightarrow \quad y^{\prime}=y+\dot{f}
$$

onde $f$ é uma função arbitrária do tempo [8]. Ou seja, pelo ponto de vista que é tradicionalmente conhecido, são justamente estas transformações que nos permitem caracterizar o sistema modelado por (14) como uma teoria clássica de calibre: pois, seja qual for a $f(t)$ que escolhemos para fixar uma solução $x(t)$, a física do sistema continua sendo descrita por um conjunto de equações que se mantém invariantes.

\subsection{Teorias de calibre como sistemas hamiltonianos com vínculos}

Apesar desse sistema, que é modelado por (14), ser um bom exemplo de uma teoria clássica de calibre onde figuram vínculos de primeira classe, ainda não explicamos como a presença destes vínculos caracteriza tal teoria. A melhor maneira de explicarmos ao leitor por que isso acontece é analisando a dinâmica de um sistema clássico com vínculos usando (10) e levando em conta algo que, conforme já dissemos na Seção 3 tem uma grande relevância: que fibrados cotangentes são exemplos de variedades [5]. Afinal, como isso implica que a parametrização $z$ adotada para $T^{*} \mathcal{M}_{n}$ não é única, isso garante que é possível tomar um outro conjunto de parâmetros para descrever um sistema clássico com vínculos através de

$$
\dot{\kappa}=\left\{\kappa, H_{\mathrm{T}}^{\prime}(\kappa)\right\}_{\Phi^{\prime}=0} .
$$

Aqui, $\Phi_{a}^{\prime}(\kappa)=0$ são os novos vínculos e $H_{\mathrm{T}}^{\prime}: T^{*} \mathcal{M}_{n} \rightarrow$ $\mathbb{R}$ é a nova hamiltoniana total que descrevem o sistema, todos adaptados a essa nova parametrização $\kappa$.

Por efeito desta linha de raciocínio, como o fato de reconhecer $T^{*} \mathcal{M}_{n-m}$ como uma subvariedade de $T^{*} \mathcal{M}_{n}$ está diretamente relacionado ao fato da decomposição diferenciável 6 ]

$$
T_{\mathrm{q}}^{*} \mathcal{M}_{n}=T_{\mathrm{q}}^{*} \mathcal{M}_{n-m} \oplus\left(T_{\mathrm{q}}^{*} \mathcal{M}_{n-m}\right)^{\perp}
$$

valer em qualquer ponto $\mathrm{q} \in \mathcal{M}_{n-m}$, onde $\left(T_{\mathrm{q}}^{*} \mathcal{M}_{n-m}\right)^{\perp}$ é o complemento ortogonal de $T_{\mathrm{q}}^{*} \mathcal{M}_{n-m}{ }^{7}$ um conjunto de parâmetros $\kappa=(\omega, \Omega)$ acaba se tornando especial: aquele onde $\omega=(q, p)$ e $\Omega=(\mathcal{Q}, \mathcal{P})$ são parametrizações intrínsecas apenas de $T_{\mathrm{q}}^{*} \mathcal{M}_{n-m}$ e de $\left(T_{\mathrm{q}}^{*} \mathcal{M}_{n-m}\right)^{\perp}$ respectivamente. A principal razão para afirmarmos que essa

\footnotetext{
${ }^{7} \mathrm{O}$ qual também pode ser chamado de espaço normal da imersão no ponto q 6].
} 
nova parametrização $\kappa$ é especial é que, independente de quaisquer considerações e/ou interpretações geométricas, já ficou demonstrado na Ref. [13 que existe uma parametrização $\kappa^{\prime}=\left(\omega^{\prime}, \Omega^{\prime}\right)$ onde uma nova hamiltoniana total

$$
H_{\mathrm{T}}\left(\kappa^{\prime}\right)=H_{\mathrm{F}}\left(\omega^{\prime}\right)+\lambda_{\mathcal{P}^{\prime}} \mathcal{P}^{\prime}+\mathcal{O}\left(\dot{\mathcal{P}}^{\prime}, \mathcal{P}^{\prime 2}\right)
$$

para um sistema clássico com vínculos pode ser expressa usando

(a) um par de variáveis canonicamente conjugadas $\omega^{\prime}=\left(q^{\prime}, p^{\prime}\right)^{8}$ que parametriza apenas $T^{*} \mathcal{M}_{n-m} \subset$ $T^{*} \mathcal{M}_{n}, \mathrm{e}$

(b) um outro par de variáveis $\Omega^{\prime}=\left(\mathcal{Q}^{\prime}, \mathcal{P}^{\prime}\right)$ que também são canonicamente conjugadas, para as quais existe uma bijeção entre as componentes de $\mathcal{P}^{\prime}=\left(\mathcal{P}_{\text {I }}^{\prime}, \mathcal{P}_{\text {II }}^{\prime}\right)$ e as de $\Phi=\left(\Phi_{1}, \ldots, \Phi_{m}\right)$.

Ou seja, ao compararmos essas duas parametrizações $\kappa$ e $\kappa^{\prime}$, podemos concluir que, na verdade, elas são as mesmas.

O aspecto interessante que segue desta conclusão é que, quando desenvolvemos (18), as equações de movimento do sistema clássico que está sob consideração se reduzem a 8

$\dot{\omega}=\left\{\omega, H_{\mathrm{F}}\right\}, \quad \dot{\mathcal{Q}}_{\mathrm{I}}=\lambda_{\mathcal{P}_{\mathrm{I}}}, \quad \dot{\mathcal{Q}}_{\mathrm{II}}=\mathcal{A}(\omega, \mathcal{Q}) \quad$ e $\quad \mathcal{P}=0$

onde: $H_{\mathrm{F}}: T^{*} \mathcal{M}_{n-m} \rightarrow \mathbb{R}$ é o que podemos chamar de hamiltoniana física; e $\lambda_{\mathcal{P}_{\mathrm{I}}}$ são os novos multiplicadores de Lagrange que implementam os novos vínculos de primeira classe $\mathcal{P}_{\mathrm{I}}=0$ à nova hamiltoniana 20. E este, sim, é o aspecto mais importante que está relacionado à caracterização de uma teoria clássica de calibre: pois, como a não univocidade, dos novos multiplicadores que implementam os novos vínculos de primeira classe, nos permite fazer infinitas escolhas para solucionar

$$
\dot{\mathcal{Q}}_{\mathrm{I}}=\lambda_{\mathcal{P}_{\mathrm{I}}} \quad \text { e } \quad \dot{\mathcal{Q}}_{\mathrm{II}}=\mathcal{A}(\omega, \mathcal{Q})
$$

é todo esse aspecto "desmontado" das equações (21) que mostra que, qualquer que seja o calibre $\lambda_{\mathcal{P}_{\text {I }}}$ que fixemos para solucionar 22$]$, esta nossa escolha jamais interferirá na solução das equações físicas

$$
\dot{\omega}=\left\{\omega, H_{\mathrm{F}}\right\} .
$$

Logo, como todas as parametrizações que podemos escolher para uma variedade se relacionam (uma com a outra) através de difeomorfismos ${ }^{9}$ é possível afirmar que uma teoria clássica de calibre é muito mais do que aquela que descreve um sistema físico

- que está vinculado a uma subvariedade $\mathcal{M}_{n-m} \subset$ $\mathcal{M}_{n}$

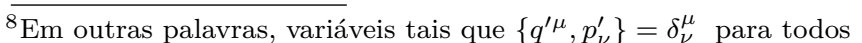
$\mu, \nu=1, \ldots, n-m$.

${ }^{9} \mathrm{Ou}$ seja, através de aplicações diferenciáveis cuja inversa também é diferenciável.
- onde o conjunto de vínculos (que define essa subvariedade $T^{*} \mathcal{M}_{n-m} \subset T^{*} \mathcal{M}_{n}$ ) é necessariamente composto pelos de primeira classe.

Como a existência de um difeomorfismo entre $z$ e $\kappa$ garante que fixar um multiplicador $\lambda_{P_{I}}$ em 21] significa fixar um multiplicador $\lambda^{\mathrm{j}}$ em 10 , esta última fixação é quem acaba definindo uma expressão

$$
\lambda^{\bar{a}}=f_{\bar{a}}(z),
$$

para todos aqueles multiplicadores de Lagrange que, por exemplo, não puderam ser determinados em (13), a qual é perfeitamente mutável. Ou seja, assim como acontece com a transformação (17), como são infinitas as escolhas dos calibres que podemos tomar para solucionar (21), também são infinitas as escolhas que temos para definir (24) (i) sem nunca afetar a solução das equações físicas do sistema e (ii) sem nunca destruir a covariância destas mesmas equações físicas, uma vez que os parênteses de Poisson são invariantes sob transformações canônicas [4].

Por se dizer, é exatamente esse aspecto que permite justificar o termo "de calibre" que está associado a essas teorias: pois, do mesmo jeito que, por exemplo, calibrar um equipamento de medida significa fazer a escolha mais conveniente (entre as várias que existem) para fixar a escala que serve como medida, fixar o calibre de um sistema clássico com vínculos significa fazer a melhor escolha que julgamos (entre as várias que existem) para a resolver as suas equações de movimento.

\subsection{O campo eletromagnético livre como exemplo de um sistema com vínculos}

Conforme já foi dito no início deste trabalho, um bom exemplo de teoria de calibre é a Eletrodinâmica Clássica: uma teoria que, por estar muito bem posta como uma teoria física, acabou servindo como um "pano de fundo" para que outras se erguessem, entre as quais podemos listar

- a Eletrodinâmica Quântica, que foi desenvolvida para descrever o comportamento quântico de sistemas eletrodinâmicos, e

- o Modelo Eletrofraco e a Cromodinâmica Quântica, que acabaram sendo agregados para definir o Modelo Padrão das partículas elementares.

Contudo, existe uma "pequena" diferença entre os exemplos que acabamos de citar e as teorias clássicas de calibre apresentadas na última Subseção: estes exemplos são modelados por funções lagrangianas

$$
L=\int_{\mathrm{V}} \mathcal{L} d \vec{x}
$$

que se valem de uma densidade $\mathcal{L}$ que depende de campos $\phi^{\mu}(x)=\phi^{\mu}(\vec{x}, t)$ e das suas derivadas, sendo $\mu=$ $0,1,2,3$ e V um volume que é definido pelas condições de contorno $\delta \phi_{\mu}(x)=0[14]$. 
Apesar das teorias clássicas de calibre com uma lagrangiana 25] serem perfeitamente interpretáveis nos mesmos moldes da Subseção anterior, a melhor maneira que existe para entendermos quais diferenças estão presentes nessa nova situação é considerando um outro exemplo simples: um, onde temos um sistema físico que, por ser composto por um único fóton, é descrito pela densidade de Maxwell

$$
\mathcal{L}=-\frac{1}{4} F_{\mu \nu} F^{\mu \nu}
$$

Aqui, $F_{\mu \nu}=\partial_{\mu} A_{\nu}-\partial_{\nu} A_{\mu}$ é a função que nos permite expressar os campos elétrico e magnético respectivamente como

$$
E_{j}=F_{j 0} \quad \text { e } \quad H_{j}=\frac{1}{2} \varepsilon_{j k l} F_{\perp}^{k l},
$$

sendo $\varepsilon_{j k l}$ o símbolo de Levi-Civita [15 onde $j=1,2,3$; ou seja,

$$
\varepsilon_{j k l}=\left\{\begin{array}{r}
1, \text { se }(j, k, l) \text { é igual a }(1,2,3) \text { ou } \\
(2,3,1) \text { ou }(3,1,2) \\
-1, \text { se }(j, k, l) \text { é igual a }(3,2,1) \text { ou } \\
(2,1,3) \text { ou }(1,3,2) \\
0, \text { caso contrário } .
\end{array}\right.
$$

\subsubsection{A presença dos vínculos de primeira classe}

De acordo com (3), ao expressarmos 26 como

$$
\mathcal{L}=\frac{1}{2}\left(\dot{A}^{j}-\partial_{j} A^{0}\right)^{2}-\frac{1}{4} F_{j k}^{2}
$$

é possível notar que os campos que correspondem aos momentos conjugados a $A^{0}$ e $A^{j}$ são dados respectivamente por

$$
P_{0}=\frac{\delta \mathcal{L}}{\delta \dot{A}^{0}}=0 \quad \text { e } \quad P_{j}=\frac{\delta \mathcal{L}}{\delta \dot{A}^{j}}=\dot{A}^{j}-\partial_{j} A^{0} .
$$

Logo, como isso mostra que não é possível expressar $\dot{A}^{0}$ em função dos campos $A=\left(A^{0}(x), \vec{A}(x)\right)$ e/ou $P=$ $\left(P_{0}(x), \vec{P}(x)\right)$, torna-se inteiramente válido interpretar

$$
\mathcal{I}_{1}=P_{0}=0
$$

como um vínculo primário, de onde segue que o formalismo hamiltoniano deste sistema eletrodinâmico pode ser primariamente definido por 8

$$
\mathcal{H}^{(1)}=\mathcal{H}+\lambda^{1} \mathcal{I}_{1},
$$

Aqui, $\lambda^{1}$ é o multiplicador de Lagrange que é necessário para implementar este vínculo primário à densidade

$$
\mathcal{H}=\frac{1}{2} P_{j}^{2}-P_{j} \partial_{j} A^{0}+\frac{1}{4} F_{j k}^{2}
$$

Certamente uma das diferenças que o leitor já deve ter notado nas últimas linhas é que, assim como já aconteceu em (15), ao invés de lidarmos com as derivadas parciais para definir os momentos conjugados, estamos lidando com derivadas parciais que são um pouco diferentes: tratam-se das derivadas parciais funcionais [9], uma vez que, assim como a ação (2), a densidade de Maxwell (26) não é uma função, mas, sim, um funciona $\sqrt{10}$. Por efeito disso, torna-se claro que, se quisermos continuar lidando com toda a estrutura que os parênteses de Poisson oferecem para analisar o nosso sistema eletrodinâmico, precisamos reescrevê-los em termos destas derivadas parciais funcionais. Nesse caso, para dois funcionais $\mathcal{F}$ e $\mathcal{G}$ que dependem dos campos conjugados $\phi^{\mu}(x)$ e $P_{\mu}(x)$, os parênteses de Poisson entre eles são definidos por

$$
\{\mathcal{F}, \mathcal{G}\}=\int\left[\frac{\delta_{t} \mathcal{F}}{\delta \phi^{\mu}(x)} \frac{\delta_{t} \mathcal{G}}{\delta P_{\mu}(x)}-\frac{\delta_{t} \mathcal{G}}{\delta \phi^{\mu}(x)} \frac{\delta_{t} \mathcal{F}}{\delta P_{\mu}(x)}\right] d \vec{x}
$$

em que $t$ está sendo usado aqui como um índice apenas para destacar o fato de que $\mathcal{F}$ e $\mathcal{G}$ precisam ser fixados no mesmo instante de tempo. Note que, pelo ponto de vista das parametrizações, é possível dizer que, nessa nova situação de um sistema clássico descrito em termos de campos conjugados, estes se encarregam de parametrizar uma espécie de "variedade funcional" onde tal sistema está definido.

Desta maneira, dando continuidade às buscas por novos vínculos com o auxílo dos "novos" parênteses de Poisson, como o vínculo primário 29 tem de satisfazer a

$$
\dot{\mathcal{I}}_{1}=\left\{\mathcal{I}_{1}, \mathcal{H}^{(1)}\right\}=0
$$

sobre a "subvariedade funcional" que ele ajuda a definir, concluímos que

$$
\mathcal{I}_{2}=\partial_{j} P_{j}=0
$$

é o único vínculo adicional que aparece. Nestes termos, como $\left\{\mathcal{I}_{1}, \mathcal{I}_{2}\right\}=0$, concluímos que 29 e (31) são dois vínculos de primeira classe e que, portanto, estamos realmente diante de uma teoria clássica de calibre.

\subsubsection{Considerações adicionais}

Apesar de tudo o que acabamos de apresentar já ser o suficiente para associar tal sistema eletrodinâmico a uma teoria clássica de calibre, não deixa de ser interessante ilustrar como equações de movimento, similares a (21), podem ser obtidas em termos de novos campos $\kappa(x)=$ $(\omega(x), \Omega(x))$, onde os pares

$$
\omega(x)=(a(x), \pi(x)) \quad \text { e } \quad \Omega(x)=(\mathcal{Q}(x), \mathcal{P}(x))
$$

satisfazem aos itens (a) e (b) respectivamente. Para que isso seja feito, além de ser importante notar que as equações desse campo eletromagnético livre são dadas por

$$
\dot{A}=\left\{A, \mathcal{H}_{\mathrm{T}}\right\}_{\mathcal{I}=0} \quad \text { e } \quad \dot{P}=\left\{P, \mathcal{H}_{\mathrm{T}}\right\}_{\mathcal{I}=0},
$$

${ }^{10} \mathrm{Ou}$ seja, a densidade lagrangiana com a qual lidamos é, como todas as outras 25, uma aplicação cujas variáveis são funções de outras variáveis. 
sendo

$$
\mathcal{H}_{\mathrm{T}}=\mathcal{H}+\lambda^{1} \mathcal{I}_{1}+\lambda^{2} \mathcal{I}_{2}
$$

a hamiltoniana total que o modela, é importante notar que, de acordo com o item (b), os conjuntos de vínculos $\mathcal{I}=\left\{\mathcal{I}_{1}(x) ; \mathcal{I}_{2}(x)\right\}$ e $\mathcal{P}=\left\{\mathcal{P}_{1}(x) ; \mathcal{P}_{2}(x)\right\}$ precisam ser equivalentes e terem o mesmo número de elementos.

Por efeito de já conhecermos o conjunto $\mathcal{I}=$ $\left\{\mathcal{I}_{1}(x) ; \mathcal{I}_{2}(x)\right\}$, cujos elementos já foram obtidos em $29 p$ e (31), não é difícil perceber que a maneira mais simples que existe para definir o novo conjunto de vínculos $\mathcal{P}$ é tomando os seus elementos como

$$
\mathcal{P}_{1}=P_{0}=0 \quad \text { e } \quad \mathcal{P}_{2}=\partial_{j} P_{j}=0 .
$$

Embora esta escolha talvez não seja a mais interessante, é diante dela e de (28) que fica claro que podemos tomar $\mathcal{Q}^{1}=A^{0}$ e $\mathcal{Q}^{2}$ como um funcional que depende de $\vec{A}(x)=\left(A^{1}(x), A^{2}(x), A^{3}(x)\right)$ : mais especificamente, de acordo com o Apêndice A, esta escolha leva a um $\mathcal{Q}^{2}=-\Delta^{-1} \partial_{j} A^{j}$, onde $\Delta^{-1}$ é o inverso do operador $\Delta=\nabla^{2}$.

Neste ponto, o leitor pode estar se perguntando por que (32) talvez não seja a escolha mais interessante para definir o conjunto $\mathcal{P}$. E a melhor resposta que podemos dar ao leitor que se faz esta pergunta é que, como o próprio Apêndice $\mathrm{A}$ também mostra que

$$
\mathcal{Q}^{\prime}=\left(A^{0}, \partial_{j} A^{j}\right) \quad \text { e } \quad \mathcal{P}^{\prime}=\left(P_{0},-\Delta^{-1} \partial_{j} P_{j}\right)=(0,0)
$$

é uma outra escolha possível, é exatamente esta outra escolha que acaba mostrando o grande argumento que podemos usar para explicar a caracterização usual desse sistema eletromagnético como uma teoria clássica de calibre. Afinal de contas, como sabemos, das equações 222, que o par $\mathcal{Q}^{\prime}=\left(A^{0}, \partial_{j} A^{j}\right)$ pode ser arbitrariamente fixado sem nunca afetar nem a solução nem a covariância das equações que descrevem o sistema físico, a escolha mais simples

$$
A^{0}=0 \quad \text { e } \quad \partial_{j} A^{j}=0
$$

que podemos fazer para resolver $\mathcal{Q}^{\prime}$ já corresponde à mesma fixação de calibre transverso [16] que é usualmente adotada quando nenhuma fonte eletromagnética se faz presente [1]. Todavia, o aspecto ainda mais interessante por trás desta fixação de calibre se relaciona justamente para com a transformação

$$
A^{\mu} \rightarrow A^{\prime \mu}=A^{\mu}-\partial^{\mu} \chi
$$

pois, como $\nabla^{2} \chi=0$, esta transformação (que a maioria dos livros apresenta como a caracterizadora da Eletrodinâmica Clássica como uma teoria de calibre) é justamente a transformação que podemos fazer sobre os campos de calibre $A^{\mu}=\left(A^{0}, \vec{A}\right)$ de modo a preservar conjugação canônica entre os campos $\mathcal{Q}^{\prime}$ e $\mathcal{P}^{\prime}$ que constam em (33).

Em todo caso, independente de qualquer predileção por $\Omega(x)=(\mathcal{Q}(x), \mathcal{P}(x))$ ou $\Omega(x)=\left(\mathcal{Q}^{\prime}(x), \mathcal{P}^{\prime}(x)\right)$, é notável que a única coisa que falta, para completar a formulação hamiltoniana em termos dos campos que satisfazem aos itens (a) e (b), é encontrar uma expressão para os campos intrínsecos $\omega(x)=(a(x), \pi(x))$. Com essa proposta em mente, e tendo ciência de todo o aspecto transverso que está embutido em (34), ao notarmos que as componentes transversas de $\vec{A}$ são expressas como

$$
A_{\perp}^{j}=\left(\delta_{k}^{j}+\Delta^{-1} \partial^{j} \partial_{k}\right) A^{j}
$$

é possível perceber que $a(x)=\left(A_{\perp}^{1}(x), A_{\perp}^{2}(x)\right)$ pode ser tomado como parte desses campos intrínsecos, uma vez que

$$
\partial_{j} A_{\perp}^{j}=0 \Rightarrow A_{\perp}^{3}=-\partial_{3}^{-1}\left(\partial_{1} A_{\perp}^{1}+\partial_{2} A_{\perp}^{2}\right) .
$$

Logo, como o campo $a(x)$ precisa ser canonicamente conjugado a $\pi(x)$, torna-se válido tomar $\pi(x)=$ $\left(P_{1}^{\perp}(x), P_{2}^{\perp}(x)\right)$, haja vista que as suas componentes

$$
P_{j}^{\perp}=\left(\delta_{j}^{k}+\Delta^{-1} \partial^{k} \partial_{j}\right) P_{j}
$$

também satisfazem a uma relação similar a (37).

Com base nestes resultados, além de ser possível demonstrar que equações do sistema físico podem ser reduzidas a 8

$$
\dot{A}_{\perp}^{\xi}=\left\{A_{\perp}^{\xi}, \mathcal{H}_{\mathrm{F}}\right\}=P_{\xi}^{\perp} \quad \text { e } \quad \dot{P}_{\xi}^{\perp}=\left\{P_{\xi}^{\perp}, \mathcal{H}_{\mathrm{F}}\right\}=\Delta A_{\perp}^{\xi},
$$

onde $\xi=1,2$ e

$$
\mathcal{H}_{\mathrm{F}}=\frac{1}{2}\left[\left(E_{\xi}^{\perp}\right)^{2}+H_{\xi}^{2}\right]
$$

é a hamiltoniana física do sistema, também é possível afirmar (através de uma "segunda maneira") que, como a solução das demais equações (não físicas) [8]

$$
\mathcal{P}_{1}=\mathcal{P}_{2}=0, \quad \dot{\mathcal{Q}}^{1}=\lambda_{\mathcal{P}_{1}} \quad \text { e } \quad \dot{\mathcal{Q}}^{2}=-\Delta \mathcal{Q}^{1}
$$

não afeta nem a solução nem a covariância das equações físicas (39), estamos realmente diante de uma teoria clássica de calibre.

\section{Comentários finais}

Como já deve ter ficado claro de tudo o que apresentamos acima, a proposta deste trabalho foi apenas a de oferecer ao leitor um texto (que tentou ser o mais didático possível dentro dos padrões de um artigo científico) com uma interpretação um pouco mais fundamental sobre o que é uma teoria clássica de calibre, sob o uso de algumas considerações geométricas e da formulação hamiltoniana de um sistema clássico com vínculos.

Existem várias referências sobre a interpretação das teorias clássicas de calibre como teorias de sistemas com vínculos. Ao leitor que se interessa por esse assunto, podemos apontar as Refs. [8] e [17], por exemplo, de onde é possível obter informações bem mais específicas e 
avançadas sobre tudo o que apresentamos aqui. Aliás, por falar nestas referências, não deixa de ser válido afirmar ao leitor que tudo o que apresentamos ao longo deste nosso trabalho pode ser interpretado como uma análise geométrica bastante simples do que consta, por exemplo, na Ref. 8].

\section{Agradecimentos}

Este trabalho foi financiado pela CAPES (ProEx) e pelo CNPq (processo número 162117/2015-9). Agradecemos a J. L. M. Assirati, A. F. Morais, S. R. A. Salinas e ao revisor da RBEF (que, apesar de não sabermos o nome, avaliou este trabalho) por todas as discussões e sugestões que fizeram deste texto algo melhor de ser lido.

\section{Material suplementar}

O seguinte material suplementar está disponível online: Apêndice A: Cálculos complementares

\section{Referências}

[1] J.D. Jackson, Classical Electrodynamics (John Willey \& Sons Inc., New York 1999), 3rd ed.

[2] I.J.R. Aitchison and A.J.G Hey, Gauge Theories in Particles Physics, Volume II: Non-Abelian Gauge Theories: $Q C D$ and the Electroweak Theory (Institute of Physics Publishing, Bristol and Phyladelphia, 2004).

[3] I.J.R. Aitchison and A.J.G. Hey, Gauge Theories in Particles Physics, Volume I: From Relativistic Quantum Mechanics to QCD (Institute of Physics Publishing, Bristol and Phyladelphia, 2003).

[4] M.F.A. de Resende, Quantização da Partícula Não Relativística em Espaços Curvos como Superfícies do $\mathbb{R}^{n}$. Dissertação de Mestrado IFUSP, São Paulo, 2011.

[5] E.L. Lima, Variedades Diferenciáveis. Monografia de Matemática, IMPA, Rio de Janeiro, 1973.

[6] M.P.do Carmo, Riemannian Geometry (Birkhäuser, Boston, 1993).

[7] L.D. Landau and E.M. Lifshitz, Mechanics (Pergamon Press, New York, 1976).

[8] D.M. Gitman and I.V. Tyutin, Quantization of Fields with Constraints (Springer-Verlag, Berlin Heidelberg, 1990).

[9] N.A. Lemos, Mecânica Analítica - Segunda Edição (Editora Livraria da Física, São Paulo, 2007).

[10] E.L. Lima, Análise Real, Volume II (Coleção Matemática Universitária IMPA, Rio de Janeiro, 2007).

[11] P.A.M. Dirac, Lectures on Quantum Mechanics (Yeshiva University Press, New York, 1964).

[12] K. Hoffman and R.A. Kunze, Linear Algebra (PrenticeHall, New Delhi, 1967).

[13] D.M. Gitman, I.V. Tyutin and Y.S. Prager, Soviet Phys. Journ. 26, 760 (1983).

[14] F. Mandl and G. Shaw G, Quantum Field Theory - 2nd Edition (John Wiley \& Sons Ltd., West Sussex, 2010).

[15] J.L. Synge and A. Shild, Tensor Calculus (Dover Publications Inc., New York, 1978).
[16] L.V. Lorenz, Phil. Mag. Scr. Series 3 34, 287 (1867).

[17] M. Henneaux and C. Teitelboim, Quantization of Gauge Systems (Princeton University Press, New Jersey, 1992). 